Document downloaded from:

http://hdl.handle.net/10251/176836

This paper must be cited as:

Moliner, L.; Correcher, C.; González Martínez, AJ.; Conde, P.; Crespo, E.; Hernandez, L.; Rigla, JP.... (2013). Time reconstruction study using tubes of response backprojectors in List Mode algorithms, applied to amonolithic crystals based breast PET. IEEE. 14-18. https://doi.org/10.1109/NSSMIC.2013.6829372

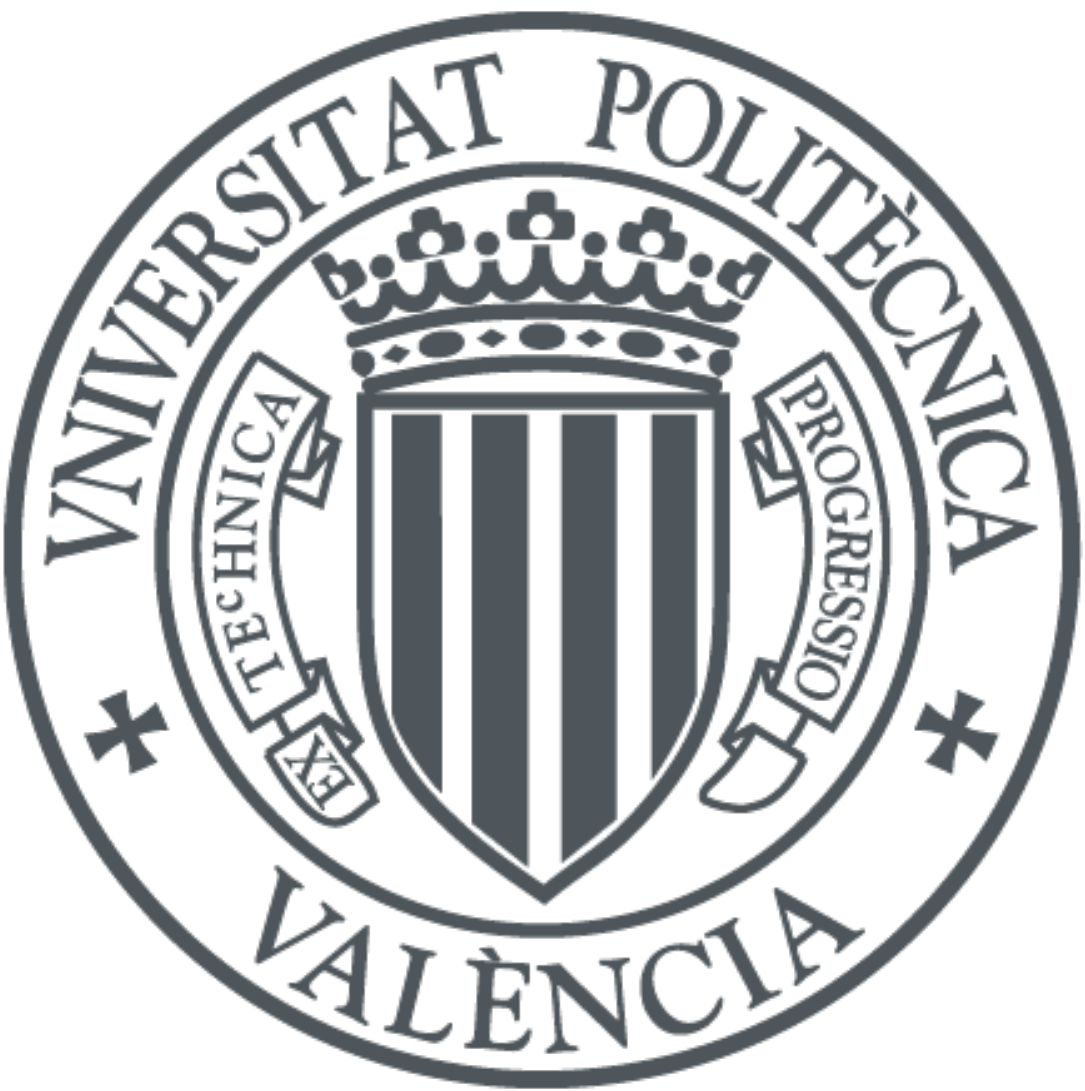

The final publication is available at

https://doi.org/10.1109/NSSMIC.2013.6829372

Copyright IEEE

Additional Information 


\title{
Time reconstruction study using tubes of response backprojectors in List Mode algorithms, applied to a monolithic crystals based breast PET
}

\author{
L. Moliner*, C. Correcher, A. J. González, P. Conde, E. Crespo, L. Hernández, J. P. Rigla, M. J. Rodríguez-Álvarez, \\ F. Sánchez, A. Soriano, A. Iborra, P. Bellido, M. Seimetz, L. F. Vidal, and J. M. Benlloch
}

\begin{abstract}
The LM-EM algorithm has the advantage to calculate the emission probabilities needed for the reconstruction process on the fly, without the need of a pre-calculated system matrix. The reconstruction time for this algorithm strongly depends on the used backprojector and the available statistics. This algorithm when implemented in systems using monolithic crystals to detect gamma radiation allows one to extensively exploit the virtual pixilation feature, not available for systems based on pixilated crystals. In this work we present a backprojector for LM-EM, the TOR method, which achieves a tradeoff between computational efficiency and image quality. Its temporal subset algorithm optimization (LM-OS) has also been implemented in order to achieve real-time reconstructions. To evaluate the performances of LM-OS algorithm with the TOR method backprojector and only with one iteration on the datasets, studies based on the system spatial resolution, uniformity, and contrast coefficients were carried out and they were compared with those obtained with LM-EM and MLEM algorithms using twelve iteration. Finally, a study on reconstruction time using $\mathrm{LM}-\mathrm{OS}$ has been performed with breast patients data.
\end{abstract}

\section{INTRODUCTION}

$\mathrm{B}_{\mathrm{E}}^{\mathrm{R}}$ REAST cancer is the major cause of cancer death in European women [1][2] as well as the most diagnosed prominent form of this type of disease. Several studies report a decline in breast cancer mortality in most European countries during the last two decades [3][4]. This decline has been attributed to the combined effect of earlier detection and improvements in therapy treatment. X-ray mammography is the most used technique for this lesions being a useful analysis tool but this, in turn presents some limitations in the examination of dense breasts [5]. Image interpretation is also subject to considerable inter-observer variability [6].

Manuscript received November 15, 2013. Project funded by the Spanish Ministry of Economy and Competitiveness and co-funded with FEDER's funds within the INNPACTO 2011 program. This work was supported by the Spanish Plan Nacional de Investigación Científica, Desarrollo e Innovación Tecnológica (I+D+i) under Grant No. FIS2010-21216-CO2-01 and the Valencian Local Government under Grants PROMETEOII/2013/010 and ISIC 2011/013.

L. Moliner,, A. J. González, P. Conde, E. Crespo, L. Hernández, J. P. Rigla, M. J. Rodriguez-Álvarez, F. Sánchez, A. Soriano, A. Iborra, P. Bellido, M. Seimetz, L. F. Vidal, and J. M. Benlloch are with Instituto de Instrumentaci'on para Imagen Molecular (I3M), CSIC -Universidad Politécnica de Valencia - CIEMAT, Camino de Vera s/n, Ed. 8-N-1, 46022 Valencia, Spain.

C. Correcher is with Oncovision. C/ Eduardo Primo Yufera, 3. 46013 Valencia, Spain.

* Corresponding author. Contact: lmoliner@i3m.upv.es
Positron Emission Tomography (PET) is a noninvasive technology that provides the user with functional images. This technique can be used in diagnosis, assessing therapy response, staging, and restaging of breast cancer [7-9]. Current whole body PET (WB-PET) systems are used for these purpose and the reconstructed images have a typical spatial resolution of about 5-15 $\mathrm{mm}$ depending on the intrinsic resolution of the scanner, imaging time, the injected dose and, the post-reconstruction image filtering applied. WB-PET achieves a detection rates higher than $92 \%$ for tumors larger than $2 \mathrm{~cm} \mathrm{[10].} \mathrm{However,} \mathrm{they} \mathrm{are} \mathrm{hardly} \mathrm{able} \mathrm{to} \mathrm{detect} \mathrm{small}$ lesions $(<1 \mathrm{~cm})$ as well as lesions with low tracer uptake [11]. Ideally, cancer diseases should be treated in the early stages of the disease [11] so, in order to overcome the above mentioned drawbacks of WB-PET, several groups are working on dedicated imaging systems based on high resolution detectors that can be placed close to the breast [12-18]. Dedicated breast PET cameras are able to provide better spatial resolution and count sensitivity than WB-PET, due to their reduced dimensions and their proximity to the source.

\section{MATERIALS}

Our group has recently developed a dedicated breast PET scanner (MAMMI) which is formed by twelve detector modules mounted on a ring configuration [19][20]. Every detector block mainly consists of a pyramidal truncated LYSO monolithic scintillation crystal coupled to a position sensitive photomultiplier tube (PSPMT). With monolithic crystals, the scintillation light distribution is preserved allowing us for an accurate photon impact determination, including its depth of interaction [21]. All crystal surfaces but the one in contact with the PSPMT are black painted in order to avoid unwanted reflections. This system has a transaxial Field of View (FOV) of $170 \mathrm{~mm}$ in diameter, and a $40 \mathrm{~mm}$ long axial FOV that axially translates to cover up to $170 \mathrm{~mm}$. The exploration is carried out in prone position without breast compression for a more comfortable patient position but specially allowing the distinction of multifocal tumors and those close to the chest walls. The data is acquired in 3D mode and later stored in list mode format. The considered coincidences are one to seven, one module with its seven opposite detectors, achieving a total of 42 pairs.

This system has implemented the maximum likelihood expectation maximization (MLEM) algorithm [22], one of the most standardized reconstruction algorithm used in PET. 
However, when MLEM or its accelerated version, ordered subset expectation maximization (OSEM) [23], are considered, the virtual pixelization on the monolithic crystals cannot be totally exploited due to the need of an storage system matrix that restricts the minimum size of virtual pixels. For this reason, the list mode expectation maximization (LMEM) algorithm [24] was implemented in the MAMMI prototype. This algorithm computes the intersection image elements for each line of response (LOR) or tube of response (TOR) and their associated weights on the fly and, therefore, a pre-calculated system matrix is no longer required. Reconstruction times using LM-EM algorithm strongly depend on the used backprojector that calculates the probabilities and the available statistics to reconstruct the acquired data. In order to achieve a tradeoff between computational efficiency and image quality the backprojector TOR method [25] based on volumetric considerations was used to calculate the voxel weights and to improve the reconstruction times. A temporal subset reconstruction using LM-OS [26] has also been implemented. In this work we propose the number of subset for the LM-OS algorithm based on studies of spatial resolution and uniformity. After the selection of this parameter, the LM-OS reconstruction is tested with studies based on image quality and patient data. Finally, a temporal study is carried on real patient data.

\section{METHOD TOR BACKPROJECTOR}

The ideal geometric backprojector will provide a list of image elements which have been crossed by a particular LOR (or TOR) and it will assign the intersection volume inside them as its own emission probability. One of the most popular approaches is the Siddon method, which assigns the crossed LOR segment inside of each voxel to its own probability. However, a coincidence between detectors could be more accurately modeled if the detectors would be linked using the TOR backprojector.

The TOR method considers the image space as intersection volumes of orthogonal sets of parallel planes. TORs are defined by a coincidence volume connecting two detector pixels, so they are cuboids crossing the image space which is formed by voxels. In our approach, all intersections will be approximated to squared areas that coincide with the dimensions of the chosen virtual pixel.

For each detected coincidence, we consider the central point of the TOR to trace a line between the involved pixels. We compute the intersection point between this line and the nearest plane formed by the voxel faces. Here, knowing the area of the TOR we can obtain the INIT and END points on the image plane, as shown in Fig. 1. These ones concern to the intersection points between the edges of the TOR and the image space. We refer the points INIT and END to its own reference system and the crossed area by the TOR inside the voxel which contains INIT and END points is calculated. Finally, using these coordinates and knowing the voxel indexes involved in the intersection we can further compute all the voxel areas. In a volumetric approximation, the product of these area values for every voxel times the length of the TOR path for the central point between two consecutive planes will be performed.
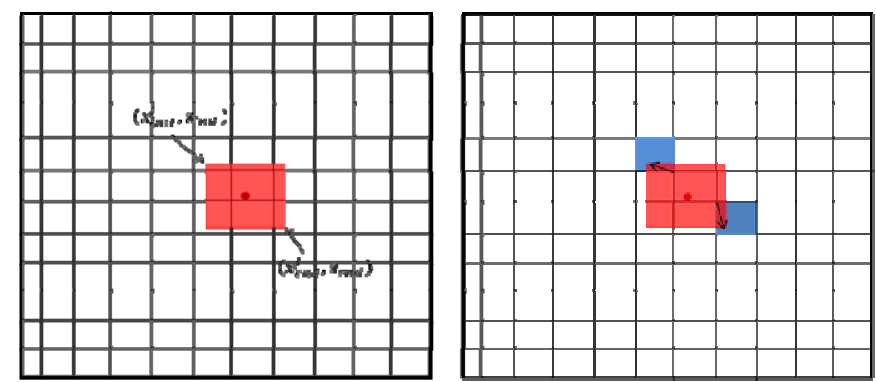

Fig. 1.Intersection between TOR and a plane formed by the faces of the voxels in the image space (left). Voxel coordinates referred to its own axis (right).

\section{MEASUREMENTS AND RESUlts}

The MLEM algorithm uses the solid angle approximation [28] as a backprojector, while LM algorithms have used the TOR method backprojector. MLEM and LM-EM algorithms have been used to reconstruct the acquired data using twelve iterations, while with LM-OS only one iteration was enabled. LM algorithms made use of virtual pixel sizes of $(1.6 \times 1.6)$ $\mathrm{mm}^{2}$ and voxel sizes of $(1 \times 1 \times 1) \mathrm{mm}^{3}$ and $(0.5 \times 0.5 \times 0.5)$ $\mathrm{mm}^{3}$, while MLEM has a fixed pixel size of $(2 \times 2) \mathrm{mm}^{2}$ and a voxel size of $(1 \times 1 \times 1) \mathrm{mm}^{3}$ due to storage limitations.

All the acquisitions were reconstructed for a different number of subsets with LM-OS. The chosen subsets varied from 20 to 100 in steps of 10 , and from 3 to 20 in steps of 1 subset. In order to simplify the notation, we will refer to the voxel dimensions by means of the " $v$ " letter followed by its size in $\mathrm{mm}$. The same notation is considered by the pixels using the "b" (bin) letter also followed by its dimensions in $\mathrm{mm}$. Data corrections such as random, scatter and attenuation have not been considered in the presented results.

\section{A. Uniformity}

The uniformity has been evaluated by means of the percentage of the standard deviation to the mean ratio for a given volume of interest (VOI). For this purpose, a cylindrical phantom was specially designed and placed at the center of the transaxial field of view (FOV) and covering the entire axial FOV. It has $40 \mathrm{~mm}$ height and $100 \mathrm{~mm}$ in diameter. The initial activity was $43 \mathrm{kBq} / \mathrm{ml}$ and the acquisition lasted 10 minutes. The considered VOI covered the $60 \%$ of the total phantom volume.

Fig. 2 shows the values of uniformity as a function of the number of subsets used by LM-OS. The algorithm starts with a smooth image estimation where all voxels values are equal to one and these values are modified as the number of subiterations increases. Therefore, the uniformity values are smaller at the beginning of the reconstruction process. Most likely, due to the noise propagation, the uniformity seems to never achieve the convergence. The v1b1.6 curve under study for LM-OS reaches the value of $18.5 \%$ at the 12-th subset which coincides with the v1b1.6 LM-EM (showed as a line). It should be noted, that from this point ahead there is a change in 
the curve slope. When 12 subsets are considered, the v05b1.6 curve $(9.32 \%)$ has the same value than the v05b1.6 LM-EM (also showed as a line), but here the change in the slope is not significantly observed.

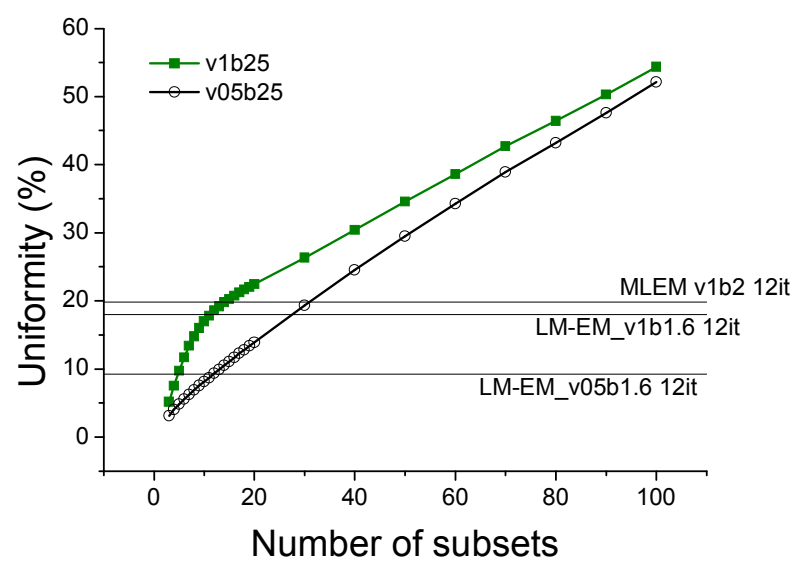

Fig. 2. Values of uniformity in $\%$ for different subset sizes with LM-OS algorithm.

\section{B. Spatial Resolution}

This spatial resolution was studied by means of the volumetric FWHM resolution of a reconstructed $37 \mathrm{kBq}{ }^{22} \mathrm{Na}$ source of $1 \mathrm{~mm}$ in diameter. This source was placed in two different positions of the transaxial FOV (center and $70 \mathrm{~mm}$ offset) and centered at the axial FOV. The acquisition time at each position was 5 minutes.

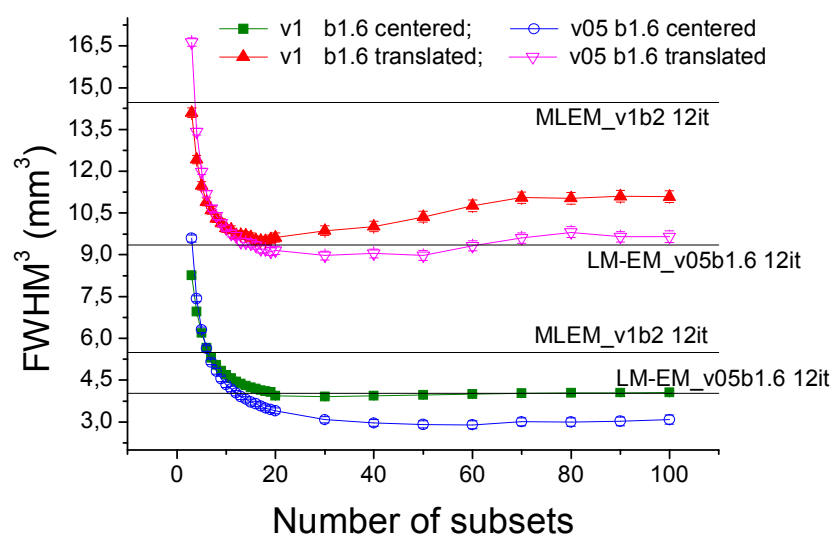

Fig. 3. Volumetric spatial resolution using LM-OS with different subsets size.

In Fig. 3 we have compared the volumetric resolutions of LMOS (using one iteration) for a different number of subsets with LM-EM and MLEM (using twelve iterations) at the two different source positions. The convergence is achieved by the source placed in the center when 20 subsets are considered. In the translated position this convergence is achieved with 12 subsets. Since the objective is to preserve a tradeoff between uniformity and spatial resolution, the obtained results suggest to consider 12 subsets for LM-OS reconstructions.

\section{Contrast Coefficients}

In order evaluate the image quality we have measured the contrast coefficients in a custom cylindrical phantom (see Fig. 4) reproducing several hot and cold lesions. This phantom has four cylindrical inserts placed $30 \mathrm{~mm}$ away from the center of the phantom. These wells were filled with different activity concentrations to model hot and cold lesions. The background region was filled with a warm activity concentration of 6 $\mathrm{kBq} / \mathrm{ml}$. The cold insert was $26 \mathrm{~mm}$ in diameter and filled with a non radiactive solution. Two of the hot lesions had a size of $20 \mathrm{~mm}$ in diameter and were filled with an activity concentration about eight and four times higher than the background activity, respectively. The third one was $15 \mathrm{~mm}$ in diameter and was filled with and activity concentration eight times higher than the background activity.

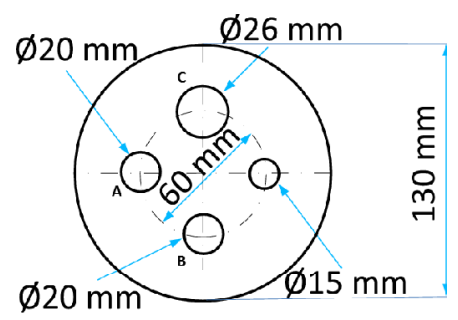

Fig. 4. Phantom designed to evaluate the image quality. .

We determined the contrast coefficients (CC) calculating the activity ratio of a VOI that covers the $80 \%$ of the total considered insert over the background divided by the real measured activity ratio [29][30], as follows:

$$
C C=\frac{(\text { measured_insert_Activity } / \text { measuredBackground })}{(\text { real_insert_Activity } / \text { realBackground })}
$$

The background VOIs were centered in the phantom with identical dimensions to the particular insert VOI. We have reconstructed the acquired data using LM-OS with 12 subsets and we have calculated the $\mathrm{CCs}$ for each insert. The results have been compared with LM-EM with 12 iterations, as shown in Fig. 5. Here, $\mathrm{SH}, \mathrm{BH}, \mathrm{BM}$ and $\mathrm{C}$ stand for Small Hot, Big Hot, Medium Hot and Cold inserts, respectively. The hot values are better when are closed to one as we can deduce from the prior equation, and it can be observed that the results obtained with LM-OS are comparable to LM-EM. However, cold region values work contrary to the hot regions and as can be seen, the LM-EM value is clearly better than LM-OS. 


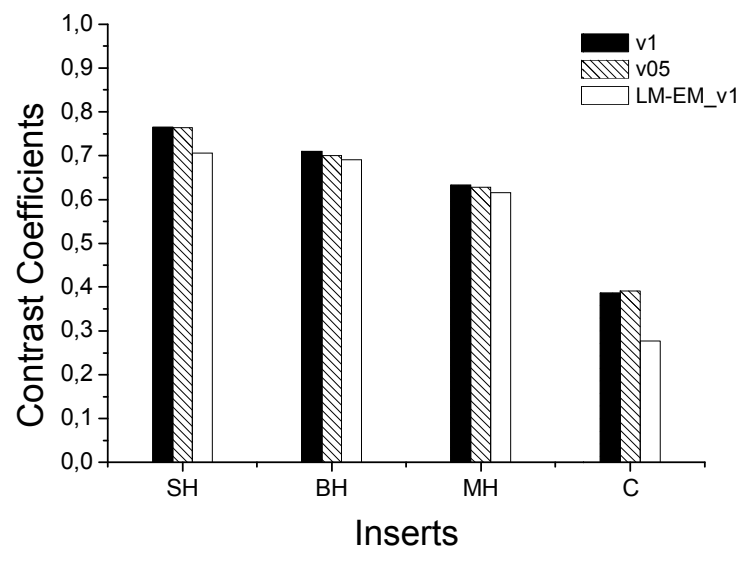

Fig. 5. Contrast Coefficients for LM-EM v1 and LM-OS with v1 and v0.5.

\section{Time studies with breast data}

In this section a time study with breast data acquisitions has been performed. Some of the acquisition parameters are listed in Table I and the reconstructed images for Patient1 are shown in Fig. 6.

\section{TABLE I. REAL PATIENT DATA}

\begin{tabular}{lccc} 
Patient & $\begin{array}{r}\text { Activity } \\
(\mathrm{MBq})\end{array}$ & $\begin{array}{c}\text { Acq. Time } \\
(\mathrm{min})\end{array}$ & $\begin{array}{c}\text { File Size } \\
\text { (coincidences) }\end{array}$ \\
\hline P1 & 81.26 & 18 & 12700000 \\
P2 & 88.65 & 20 & 11000000 \\
P3 & 85.00 & 20 & 8000000 \\
\hline
\end{tabular}

The algorithms under study have been coded in parallel to take advantage of the latest computational multi-core architectures. The forward and backprojection processes have been parallelized distributing LORs and voxels in the different processors, respectively. Moreover, the image update and synchronization have been made with a single processor. In this work, a dual Intel Xeon processor computer with eight cores and 12GB RAM memory was used. All the coding has been implemented in 64 bits. A comparative study of reconstruction times for all the implemented algorithms is plottedinFig. 7.


Fig. 6. Breast reconstructed images with different algorithms. Top-left LMOS_v05b1.6, top-right MLEM_v1b2, bottom-left LM-OS_v1b1.6 and bottomright LM-EM_v1b1.6.

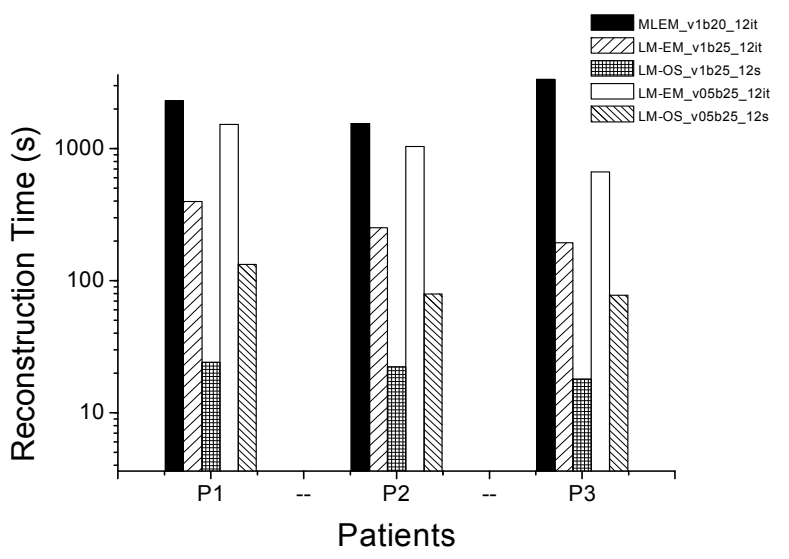

Fig. 7. Reconstruction times for breast acquisitions using MLEM, LMEM_v1b1.6, LM-EM_v05b1.6, LM-OS_v1b1.6 and LM-OS_v05b1.6.

The reconstruction times using LM-OS_v05b1.6, where the image quality shows the best results, are around 130 seconds whereas using LM-OS_v1b1.6 the reconstruction only takes 30 seconds.

\section{CONCLUSIONS}

Twelve subsets when using the LM-OS algorithm (using one iteration) for the MAMMI breast PET system have been decided, based on spatial resolution and uniformity studies. The results for contrast coefficients are similar to those obtained with LM-EM (using twelve iterations), but the cold region would improve when enabling the attenuation correction to achieve the same values than LM-EM.

The time reconstruction results suggest that since the typical acquisition times with the MAMMI prototype are around 20 min, it can be stated that the algorithm achieves the real time reconstruction with daily patient explorations.

\section{ACKNOWLEDGMENT}

This work was supported by the Spanish Plan Nacional de Investigación Científica, Desarrollo e Innovación Tecnológica (I+D+I) under Grant No.FIS2010-21216-CO2-01 and Valencian Local Government under Grants PROMETEOII 2013/010 and ISIC 2011/013.

\section{REFERENCES}

[1] F J. Ferlay, P. Autier, M. Boniol, M. Heanue, M. Colombet, and P. Boyle, "Estimates of cancer incidence and mortality in Europe in 2006," Ann.Oncol. 18, 581-592 (2007).

[2] J. Ferlay, D. M. Parkin, and E. Steliarova-Foucher, "Estimates of cancer incidence and mortality in Europe in 2008," Eur. J. Cancer 46, 765-781 (2010).

[3] F. Levi, C. Brosetti, F. Lucchini, E. Negri, and C. La Vecchia, "Monitoring the decrease in breast cancer mortality in Europe," Eur. J. Cancer Prev. 14(6), 497-502 (2005).

[4] J. E. Tyczynski, I. Plesko, T. Aareleid, M. Primic-Zakelj, M. Dalmas, J. Kurtinaitis, A. Stengrevics, and D. M. Parkin, "Breast cancer mortality patterns and time trends in 10 new EU member states: Mortality declining in young women, but still increasing in the elderly," Int. J. Cancer 112, 1056-1064 (2004).

[5] C. H. J. Chang, D. E. Nesbit, D. R. Fisher, S. L. Fritz, S. J. Dwyer III, A. W. Templeton, F. Lin, and W. R. Jewell, "Computed tomographic mammography using a conventional body scanner," Am. J. Roentgenol. $138,553-558(1982)$. 
[6] K. Kerlikowske, D. Grady, J. Barclay, S. D. Frankel, S. H. Ominsky, E. A. Sickles, and V. Ernster, "Variability and accuracy in mammographic interpretation using the American College of Radiology breast imaging reporting and data system," J. Natl. Cancer Inst. 90(23), 1801-1809 (1998).

[7] P. Lind, I. Igerc, T. Beyer, P. Rainprecht, and K. Hausegger, "Advantages and limitations of FDG-PET in the follow-up of breast cancer," Eur. J. Nucl. Med. Mol. Imaging 31(Suppl. 1), S125-S134 (2004).

[8] H. Linden, S. Stekhova, J. Link, J. Gralow, R. Livingston, G. Ellis, P. Petra, L. Peterson, E. Schubert, L. Dunnwald, K. Krohn, and D. Mankoff, "Quantitative fluoroestradiol positron emission tomography imaging predicts response to endocrine treatment in breast cancer," J. Clin. Oncol. 24(18), 2793-2799 (2006).

[9] W. Eubank and D. Mankoff, "Evolving role of positron emission tomography in breast cancer imaging," Semin Nucl. Med. 35(2), 84-99 (2005).

[10] N. Avril, C. Rosé, M. Schelling, J. Dose, W. Kuhn, S. Bense, W. Weber, S. Ziegler, H. Graeff, and M. Schwaiger, "Breast imaging with positron emission tomography and fluorine-18 fluorodeoxyglucose: Use and limitations," J. Clin. Oncol. 18(20), 3495-3502 (2000).

[11] C. L. Carter, C. Allen, and D. E. Henson, "Relation of tumor size, lymph node status, and survival in 24740 breast cancer cases," Cancer 63(1), 181-187 (1989).

[12] Y. Wu, S. Bowen, K. Yang, N. Packard, L. Fu, G. Burkett, J. Qi, J. Boone, S. Cherry, and R. Badawi, "PET characteristics of a dedicated breast PET/CT scanner prototype," Phys. Med. Biol. 54, 4273-4287 (2009).

[13] H. Li, W.-H. Wong, H. Baghaei, J. Uribe, Y. Wang, Yu Zhang, S. Kim, R. Ramirez, J. Liu, and S. Liu, "The engineering and initial results of a transformable low-cost high-resolution PET camera," IEEE Trans. Nucl. Sci. 54(5), 1583-1588 (2007).

[14] W. Luo, E. Anashkin, and C. G. Matthews, "Performance evaluation of a PEM scanner using the NEMA NU 4-2008 small animal PET standards," IEEE Trans. Nucl. Sci. 57(1), 94-103 (2010).

[15] R. R. Raylman, S. Majewski, M. F. Smith, J. Proffitt, W. Hammond, A. Srinivasan, J. McKisson, V. Popov, A. Weisenberger, C. O. Judy, B. Kross, S. Ramasubramanian, L. E. Banta, P. E. Kinahan, and K. Champley, "The positron emission mammography/tomography breast imaging and biopsy system (PEM/PET): Design, construction and phantom-based measurements," Phys. Med. Biol. 53, 637-653 (2008).

[16] A. Vandenbroucke, A. M. K. Foudray, P. D. Olcott, and C. S. Levin, "Performance characteristics of a new high resolution PET scintillation detector," Phys. Med. Biol. 55, 5895-5911 (2010)

[17] K. Kitamura, J. Ochi, H. Tonami, Y. Yamada, T. Furimiya, M. Furuta, M. Satoh, T. Tsuda, M. Nakazawa, N. Hashizume, Y. Yamakawa, A. Kawashima, and Y. Kamazawa, "Development of a C-shaped breast PET scanner equipped with four layer DOI detectors," in 2008 IEEE Nuclear Science Symposium Conference Record, MR10-2 (IEEE, Piscataway, NJ, pp. 5662-5664 (2008).

[18] B. Ravindranath, S. H. Maramraju, S. S. Junnarkar, S. S. Southekal, S. P. Stoll, J. F. Pratte, M. L. Purschke, X. Hong, D. Bennett, K. Cheng, D. Tomasi, S. Krishnamoorthy, P. Vaska, C. L. Woody, and D. J. Schlyer, "A simultaneous PET/MRI breast scanner based on the RatCAP," in 2008 IEEE Nuclear Science Symposium Conference Record,M10-56 (IEEE, Piscataway, NJ, pp. 4650-4655 (2008).

[19] J.A. González, T.S. Aukema, J. Barberá, J.M. Benlloch, C.Correcher, A. Orero, A. Soriano, W. Vogel, C. Vázquez, L.F. Vidal, R.A. Valdés, "Design and firts results of an innovative and dedicated breast PET" Curr. Med. Imag. Rev. 8, 144 (2012).

[20] L. Moliner, C. Correcher, A.J. González, A. Soriano, F. Sánchez, A. Orero, M. Carles, L.F.Vidal, J. Barbera, L. Caballero, M. Seimetz, C. Vázquez, J.M. Benlloch "Design and evaluation of the MAMMI dedicated breast PET", Med. Phys. 39, 5393-404 (2012)

[21] C. W. Lerche, A. Ros, R. Gadea, R. J. Colom, F. J. Toledo, V. Herrero, J. M. Monzó, A. Sebastiá, F. Sánchez, C. Correcher, A. J. Gonzalez, A. Munar, and J. M. Benlloch, "DOI measurement with monolithic scintillation crystals: A primary performance evaluation," in 2007 IEEE Nuclear Science Symposium Conference Record, M03-1 (IEEE, Piscataway, NJ, pp. 2594-2600 (2007).

[22] L. Sheep and Y. Vardi, "Maximum likelihood reconstruction for emission tomography," IEEE Trans. Med. Imaging MI-1(2), 113-122 (1982).
[23] H.M. Hudson, R.S. Larkin, Accelerated Image Reconstruction Using Ordered Subsets of projection Data, IEEE. Trans. Med. Img. 13, 601609 (1994)

[24] H.H. Barrett, T. White, L. C. Parra, List-mode likelihood, J. Opt. Soc. Am. A/ Vol. 14, No. 11/November, 2914-2923 (1997).

[25] L. Moliner, C. Correcher, A.J. Gonzalez, P. Conde, L. Hernandeza,, A. Orero, M.J. Rodriguez-Alvarez, F. Sanchez, A. Soriano, L.F. Vidal, J.M.Benlloch, Implementation and analysis of list mode algorithm using tubes of response on a dedicated brain and breast PET, NIM-A 720, 129$132(2013)$

[26] A.J. Reader, R.Manavaki, S. Zhao, P.J. Julyan, D.L. Hastings, J. Zweit, Accelerated List-Mode EM Algortihm, IEEE Trans. Nucl. Sc. 49, 42-49 (2002).

[27] R.L. Siddon, Fast calculation of the eact radiological path for a three dimensional CT array, Med. Phys. 12 (1985) 252-255.

[28] A.V. Oosteron, J. Strackee, The solid angle of a Plane Triangle, Trans. Bio. 187 Eng. 12, 125-126 (1983).

[29] NEMA Standards Publication NU 2-2007, Performance measurements of Positron Emission Tomographs. NEMA Standards, (2007).

[30] NEMA Standards Publication NU 4-2008, Performance measurements of Small Animal Positron Emission Tomographs. NEMA Standards, (2008). 